\title{
Powdery and Downy mildew infections regulates a cascade of defense-responsive genes in Vitis Vinifera
}

Neetu Goyal

Panjab University

Garima Bhatia

Panjab University

Naina Garewal

Panjab University

Anuradha Upadhyay

NRC Grapes, Pune

Kashmir Singh ( $\nabla$ kashmirbio@pu.ac.in )

Panjab University https://orcid.org/0000-0002-9646-9043

Research article

Keywords: Grapevine, Fungal infections, mildew resistance, Defensive genes, Expression, Salicylic acid

Posted Date: September 30th, 2019

DOI: https://doi.org/10.21203/rs.2.15331/v1

License: (9) This work is licensed under a Creative Commons Attribution 4.0 International License.

Read Full License 


\section{Abstract}

Background: Grape cultivation is severely affected by powdery mildew and downy mildew worldwide leading to significant losses in productivity and quality. To control such diseases in an environment friendly way, it is essential to understand the molecular mechanisms of fungal resistance in grapes. When the plant is infected with pathogen, various defense mechanisms are activated in plants at molecular level, the ultimate aim of all is to provide immunity against infection.

Results: In our study, we tried to understand the Salicylic acid (SA) mediated pathway of ETI through a cascade of defense molecules ultimately leading to activation and expression of PR proteins that provides SAR in plants. We performed genome-wide identification of defensive molecules expressed during PM and DM infection in grapevine. Consequently, we identified 2, 4, 7, 4, 2, 1, 20 and 7 differentially expressed PM-responsive defensive genes ( NBS-LRR, EDS1, NDR1, NPR, PAD4, RAR1, TFs and PR ) and 28, 2, 5, 4, 1, 39 and 19 differentially expressed DM-responsive defensive genes ( NBS-LRR, EDS1, NDR1, NPR, RAR1, TFs and PR ) in V. vinifera. Next, the co-expression between the identified defensive genes was performed that tells us about the sequence of events that occurs during a signaling cascade in response to PM and DM-infection. Further, we characterized the identified genes on the basis of physico-chemical properties, chromosomal positions, gene and protein structure analysis and functional annotation.

Conclusion: The powdery and downy mildew responsive defensive genes identified in this study can be used to develop new and improved PM and DM-resistant grape varieties in future.

\section{Introduction}

Grape is one of the commercially important fruit crop cultivated in India. However, the production of grapes is severely hampered due to the climate of major grape producing areas in India that allows the development of various fungal diseases. Powdery mildew (PM) and downy mildew (DM) are two such devastating diseases that require cooler temperatures and higher relative humidity to propagate and infect the grapevine [1]. The epidemic of PM and DM acutely diminishes the quality as well as productivity by infecting all green tissues of the grapevine. As most of the cultivated varieties in India belong to Vitis vinifera, which is susceptible to PM and DM, therefore, it is necessary to understand the molecular biology of powdery and downy mildew resistance mechanism in grapevine for controlling such fungal diseases.

During conditions of abiotic and biotic stresses, various defense mechanisms are activated in plant as a part of their natural immune response to protect itself. Basically, 3 major steps are involved in host defense against pathogenic attack i.e recognition of pathogens, signal transduction, and induction of genes that leads to production of molecules with antimicrobial activity. During PM and DM infection, under favorable environmental conditions, the conidiospores of Erysiphe necator (PM fungus) and Plasmopara viticola (DM fungus) germinate on the grapevine leaf surface, leading to initial interaction of 
Pathogen-associated molecular patterns (PAMPs) and grapevine defense molecules (PRRs), thus providing first layer of defense i.e Pathogen Triggered Immunity (PTI) [2] [3] However, the activated defense is not sufficiently robust to prevent the fungal proliferation in plant. Additionally, to enhance its invasion in plant, fungus delivers certain effector proteins inside the cell via different mechanisms such as Type 3 effector proteins, haustoria etc. During that time, another defense mechanism known as Effector Triggered Immunity (ETI) is activated that triggers the expression of resistance (R) proteins often resulting in apoptosis of the infected cell, thereby blocking the further proliferation of the pathogen [4] [5].

Various signal transduction pathways that are activated during ETI lead to re-programming of the host plant transcriptome and activation of defensive genes. It is believed that the resistance of plant against biotrophic pathogens is mediated through Salicylic acid (SA) signaling in which SA acts as secondary messenger [6]. As Erysiphe necator and Plasmopara viticola are both biotrophic in nature, therefore the defence mechanism activated in plant in response to mildew infection is signaled through SA. There are cascade of events that occur during this signaling and various genes get activated that acts upstream and downstream of SA. It has been reported previously that the R-gene mediated resistance response is activated via 2 key proteins i.e Enhanced Disease Susceptibility 1 (EDS1) and Non-Race-specific Disease Resistance (NDR1), both interacting with different types of R proteins. EDS1 interacts with Toll/interleukin-1 receptor (TNL) type R proteins and NDR1 interacts with Coiled coil (CNL) type $R$ proteins [7]. Recent studies demonstrated that any perturbation of the interaction between EDS1, NDR1 and $R$ proteins can affect the activation of signaling pathway thereby suggesting the importance of these 2 central regulators of plant defense response [8] [9] [10] [11]. Some other major molecules of SA mediated defense signaling includes Phytoalexin-deficient 4 (PAD4),, avrPphB susceptible 2(PBS2), Senescence associated gene 101SAG101), , required for Mla-specified resistance (RAR1), which get functionally activated upstream of SA to promote SA accumulation.

Additionally, SA signaling requires one major protein downstream of SA i.e Nonexpressor of PR Gene1 (NPR1) gene. This gene is a key regulator of SA mediated signal transduction which got activated after accumulation of SA [12]. Before activation, NPR1 is present in cytoplasm in oligomeric form, after accumulation of SA, NPR1 reduces to monomeric units that get translocated into the nucleus where it interacts with various TGA and WRKY transcription factors (TFs) and ultimately leads to expression of Pathogenesis Related (PR) genes, the final players of plant defense response [13] [6] [14].

Altogether, accumulation of SA and expression of $P R$ genes leads to development of Systemic Acquired Resistance (SAR) i.e a broad spectrum resistance in plants [15].

Previously, we characterized 63 PM-responsive NBS-LRR genes from different grapevine cultivars by analyzing transcriptome of these cultivars inoculated with $E$. necator. In this study, we investigated the interaction between various defense responsive genes activated in response to PM and DM infection and proposed a pathway for the same. Additionally, we characterized the identified genes on the basis of chromosomal locations, gene and protein structure analysis, physicochemical properties and functional 
domain annotation using BLAST2GO. Moreover, we also depicted numerous cis-acting regulatory elements (CARE) for the identified genes in response to powdery and downy mildew stress.

\section{Material And Methods}

Identification of PM and DM responsive defensive genes in V. vinifera

Protein coding sequences of various gene families i.e EDS1, NDR1, NPR1, RAR1, TFs, PR reported in grapes were extracted from National Center for Biotechnology Information (NCBI) genome database (ftp://ftp.ncbi.nlm.nih.gov/genomes/Vitis_vinifera/protein/) and grape genome browser 12X i.e genoscope (http://www.cns.fr/externe/GenomeBrowser/Vitis/) and generated a fasta file. To identify PM and DM responsive defensive genes, we downloaded the RNA-seq data of Thompson Seedless and Pinot Noir varieties of $V$. vinifera with project numbers of SRP116308 and PRJEB24540 with the use of NCBI Sequence Read Archive (SRA) (http://www.ncbi.nlm.nih.gov/sra). The transcriptomic data of Thompson Seedless was responsive to PM and was available at 1 time point i.e 36 hours post inoculation (hpi) (Northwest A\&F University). Similarly, the RNA seq data of Pinot Noir is DM-responsive and is available at 3 time points i.e 1dpi, 2dpi and 3dpi [16]. Previously, we reported the occurrence of 386 NBS-LRR genes in $V$. vinifera [17]. We used these gene sequences to identify PM and DM-responsive NBS-LRR gene sequences. The abundance of all fungal responsive defensive genes were calculated by RSEM (RNA-Seq by Expectation-Maximization) software of the Trinity-V2.4 package as fragments per kilobase of transcript per million fragments mapped (FPKM) [18]. Subsequently, EdgeR was used to measure the differential gene expression by applying the cut-off value of four folds of change in expression level and a $P$ value of 0.001 . The differential gene expressions of all classes of genes identified in our study were represented as heat maps and Hierarchical Clustering Explorer 3.5 was used to generate heat maps (http://www.cs.umd.edu/hcil/hce/) [19]. Further, on the basis of heat maps generated, the fold change and number of up and down regulated defensive genes were determined in response to powdery and downy mildew infection in $V$. vinifera.

\section{Co-expression analysis}

Next, we conducted co-expression (CE) analysis of differentially expressed defensive genes to determine the sequence of events that occurs during a signaling cascade in response to PM and DM-infection. The CoExpress 1.5.2 software tool was used with default parameters (http://Bioinformatics.lu/CoExpress). The data of two sets of genes was loaded at a time and a linear correlation was measured amongst them. Finally, we screened genes that are highly co-expressing with the R value of 1 or close to 1 . The visualization of co-expression networks for genes was done using the software cytoscape 3.7.1 (https://cytoscape.org/).

\section{Plant material and treatment}

As $\mathrm{SA}$ is playing a pivotal role in defense against fungal infections, we studied its effect on Thompson Seedless variety of $V$. vinifera. The stem cuttings were grown in pots containing soil:soil-rite in the ratio of 
2:1 in growth chamber in Department of Biotechnology, Panjab University, Chandigarh. The SA treatment was given at the final concentration of $100 \mathrm{mg} /$ litre at 5 different time intervals i.e $1 \mathrm{hpi}, 2 \mathrm{hpi}, 1 \mathrm{dpi}, 2 \mathrm{dpi}$ and $3 \mathrm{dpi}$ and subsequently the control and treated leaves were collected, snap frozen and stored in $-80^{\circ}$ $C$ till further use. Additionally, we collected the healthy and fungal infected leaf samples of Thompson Seedless variety of $V$. vinifera from National Research Centre for Grapes (NRCG), Pune.

RNA isolation, $C D N A$ synthesis and quantitative real time PCR analysis

On the basis of genes identified in co-expression analysis, we performed quantitative real time PCR (qPCR) of PM and DM-responsive differentially expressed defensive genes to measure the relative expression. Leaf samples were used to isolate the total RNA by following Ghawana et al. 2011 protocol and subsequently, the cDNA was prepared by using Superscript III first strand cDNA synthesis kit (Invitrogen USA) [20]. We used two housekeeping genes namely actin (ACT) and elongation factor 1 (EF1) as internal reference genes for the normalization of qPCR results. The real time primers were designed by using Primer 3 software (http://primer3.ut.ee/). We performed qPCR experiment with three replicates of healthy, fungal and SA treated leaf samples by employing Bio-Rad CFX96 Real-Time PCR detection system. The following conditions were used while performing an experiment: $95 \bowtie \mathrm{C}$ for $7 \mathrm{~min}$, followed by 40 cycles of $95 \otimes C$ for $20 \mathrm{sec}, 57 \llbracket \mathrm{C}$ for $20 \mathrm{sec}$ and $72 \varangle \mathrm{C}$ for $20 \mathrm{sec}$. To analyze the results of qPCR, we used REST 2009 software (Qiagen) and 2(- $\Delta \Delta \mathrm{CT})$ method to obtain relative gene expression ratios of target genes with respect to control genes (http://www.REST.de.com) [21] [22].

\section{Gene and protein structure analysis}

We determined the gene structure by identifying the exons and introns present in the gene sequences. The coding sequence of each gene was aligned to its genomic sequence using an online tool 'Splign' (http://www.ncbi.nlm.nih.gov/sutils/splign/splign.cgi). Further verification of the aligned result was done by using Ensembl Plants portal (http://plants.ensembl.org/Vitis_vinifera/Gene). The visualization of position of exons and introns was done by using GSDS2.0 server (http://gsds.cbi.pku.edu.cn/) [23]. Additionally, we plotted the intron phases for our gene sequences with the same tool. Next, the conserved protein motifs were discovered by using Multiple Expectation Maximization for Motif Elicitation (MEME) Suite (http://meme-suite.org/tools/meme) with default parameters. [24].

\section{Chromosomal mapping of PM and DM-responsive defensive genes}

To determine the positions of PM and DM-responsive defensive gene sequences chromosome-wide, chromosomal mapping was done. Primarily, the gene sequences were searched against non-redundant (nr) database by performing BLASTN and short-listed the hits depicting maximum identity and subsequently located the gene on respective chromosome. Additionally, the confirmation of chromosomal position of genes was done through Grape Genome Browser i.e. Genoscope (http://www.genoscope.cns.fr/externe/GenomeBrowser/Vitis/) and Ensembl Plants portal (https://plants.ensembl.org/Vitis_vinifera/Location/Genome). 


\section{Functional characterization}

For the functional annotation of PM and DM-responsive defensive genes, BLAST2GO (https://www.blast2go.com/blast2go-pro) tool was used [25]. Basically, 3 steps are involved in BLAST2GO annotation: BLAST for finding homologous sequences, Mapping to assign GO terms for each hit obtained, Annotation to assign function to query sequences on the basis of their cellular localizations, molecular functions and biological processes.

\section{Promoter analysis}

The cis-acting regulatory elements of PM and DM-responsive defensive genes were predicted using PlantCare database (http://bioinformatics.psb.ugent.be/webtools/plantcare/html/) [26]. We extracted a 2-kb sequence upstream of translation start site after aligning the genomic sequences with coding sequences and analyze it eventually for the presence of different regulatory elements.

\section{Physicochemical characteristics of PM and DM-responsive defensive genes}

The physicochemical characteristics of PM and DM-responsive defensive genes were depicted using ProtParam bioinformatics tool (https://web.expasy.org/protparam/), which provides the information regarding the number of amino acids, theoretical pl (isoelectric point), molecular weight and instability index of protein [27].

\section{Results And Discussion}

\section{Identification of fungal responsive defensive genes in $\mathrm{V}$. vinifera}

To better understand the molecular mechanism of powdery and downy mildew resistance in grapevine, we performed genome-wide identification of PM and DM-responsive defensive genes in grapes in response to salicylic acid mediated signal transduction pathway, the ultimate goal of which is to provide ETI in plants. By using Genoscope and NCBI, we retrieved 9, 10, 20, 4, 3, $177(47,59,71)$ and 44 EDS, NDR1, NPR, RAR1, PAD, TFs (TGA, WRKY, NAC) and PR protein coding sequences of $V$. vinifera and developed a fasta file. Additionally, we also used 386 NBS-LRR gene sequences identified in our previous study. Next, to identify fungal responsive defensive genes, normalized expression analysis of the above mentioned gene classes was conducted. To accomplish this task, we extracted the transcriptomic data of one PM-susceptible (Thompson seedless) and one DM-susceptible (Pinot Noir) V. vinifera varieties from $\mathrm{NCBI}$ and conducted the normalized expression analysis (FPKM) analysis. Consequently, we identified varied number of differentially expressed PM-responsive and DM-responsive defensive genes in $V$. vinifera. As, NBS-LRR proteins are the primary proteins recognized by avirulent proteins of the pathogen, hence, we identified 2 highly expressing PM-responsive and 28 DM-responsive NBS-LRR genes that were differentially regulated in the present study (Fig. 1). Our results showed that most of the fungal responsive NBS-LRR genes were up-regulated during later stages of fungal infection as compared to initial phase. Various fungal responsive $N B S-L R R$ genes have also been identified previously in many 
plants such as apple, Arabidopsis, wheat, grapes, Cucumber etc [28] [29] [30] [31] [32], [33] [34]. In one study conducted by Coleman et al in 2009, a cluster of NBS-LRR genes was identified in Resistance to Erysiphe necator 1 (REN1) locus that is responsive to PM in Kishmish vatkana' and 'Dzhandzhal kara' grapevine varieties [32]. Likewise, in V. amurensis and V. riparia grapevine varieties, NBS-LRR gene cluster was identified in response to DM infection.

Later on, after identification of NBS-LRR genes, our research is being directed towards identification of molecules that leads to activation of NBS-LRR proteins. In search of that, two key genes i.e EDS1 and NDR1 were identified that were showing differential expression pattern in response to PM and DMinfection. We identified 4 EDS1 and 7 NDR1 differentially expressed genes in response to PM infection; 2 EDS1 and 5 NDR1 in case of DM-infection (Fig. 1). Our results also showed that all EDS1 genes got upregulated during PM-infection as compared to control condition and out of 7, 5 NDR1 genes got upregulated during PM-infection. Similarly, during DM-infection, all genes got up-regulated during later stages (3 dpi) of infection.

Further from one study conducted in Arabidopsis, it was known that EDS1 interacts with PAD4 present downstream and both these proteins positively regulate the accumulation of SA to provide R-gene mediated resistance in plant [35]. So, to study the interaction of EDS1 and PAD4, we identified differentially expressed PAD4 genes in grape genome. As a result, we got 2 PAD4 genes through FPKM analysis in PM infected samples out of which one gene is upregulated but surprisingly, we did not get any PAD4 gene expressing in DM-infected sample (Fig. 1).

There is one another protein that is found in our study acting upstream of SA i.e RAR1, a co-chaperone of HSP90. We found 1 PM-responsive and 1 DM-responsive differentially expressed transcript encoding $R A R 1$ in case of both $\mathrm{PM}$ and $\mathrm{DM}$ infection and both the transcripts were showing up-regulation during fungal stresses (Fig. 1). Till now, all these genes lead to accumulation of SA in plant cell that is essential for local and SAR in plant.

Further, downstream of SA, another important protein activated is NPR1. This gene is considered as master regulator of SA-mediated immune response. There are reports on NPR1 gene that tells us that over-expression of this gene in Arabidopsis, wheat, tomato and apple confers broad spectrum resistance against fungal and bacterial pathogens [36] [37] [38] [39]. In our analysis, we found 4 PM-responsive and 4 DM-responsive NPR (comprising of NPR1 and NPR2) genes that got differentially expressed during fungal infection and all NPR genes were seen to be upregulated gradually from initial to later stages of both powdery and downy mildew infection (Fig. 1).

NPR1 and NPR2 further interacts with different classes of transcription factors. Through differential expression analysis, we identified 20 PM-responsive and 39 DM-responsive TFs that belongs to 3 different classes i.e bZIP, WRKY and NAC. The interaction of NPR proteins with several TFs finally activates various PR proteins that provides SAR to plant. 
The description of plant PR proteins was first made in tobacco leaves infected by tobacco mosaic virus [40]. Since then, these proteins have been identified in many monocot and dicot plants. Usually, the accumulation of PR proteins is induced in plants during conditions of pathogenic infection, thereby playing an eminent role in improving the defensive ability of plant. [41]. In our study, we found 7 PMresponsive and 19 DM-responsive differentially expressed PR proteins that belongs to 5 different classes of PR proteins i.e PR1 (Antifungal), PR2 ( $\beta-1,3-$ Glucanase), PR5 (Thaumatin-like), PR10.1 (Ribonucleaselike) and PR10.7 (Ribonuclease-like) (Fig. 1). The role of all the PR proteins identified in our study has been previously identified in various grape varieties as documented in literature. Previously, PR1 proteins exhibiting anti-fungal property has been identified and cloned in $V$. vinifera [42]. However, till date, its role in signaling pathway was not determined in grapes. Our study suggested that PR1 is activated during SA mediated signaling cascade in response to PM and DM infection in grapes. PR-2 proteins have the capacity of hydrolyzing the components of fungal cell wall, thus exhibiting antimicrobial activity. In 1999, Jacobs et al. showed that the hydrolytic activity in grape directly affects growth of powdery mildew infection at the pathogen infection site [43]. Similarly, PR-5 proteins enhance fungal membrane permeability and causing osmotic rupture of fungal plasma membrane providing immunity against fungal attack [44]. PR-10 proteins acts directly against pathogens or can contributes to plant defense in programmed cell death during HR [45]. Previously, $P R-10$ gene was isolated from $V$. pseudoreticulata that is showing resistance to $P$. viticola infection [46]. All these studies of PR proteins supported our results of PR proteins activated in response to SA in grapes.

Overall in our study, we identified 47 PM-responsive and 98 DM-responsive differentially expressed genes in $V$. vinifera accessions used in our study (Fig. 1). Overall, our results suggested that most of the genes got upregulated during PM and DM infection conditions as compared to control ones.

\section{Co-expression analysis}

Next, we conducted co-expression (CE) analysis of differentially expressed PM and DM-responsive defensive genes using the software tool CoExpress 1.5.2. Subsequently, we screened some highly coexpressing genes in $V$. vinifera that might be regulating the powdery and downy mildew infection through SA signaling cascade. The visualization of the co-expressed genes was done by using the software Cytoscape 3.7.1 with default parameters (Fig. 2). Amongst all, we got 6 PM-responsive and 7 DMresponsive NBS-LRR genes that were found to be showing strong co-interaction with EDS1 and NDR1 genes residing downstream NBS-LRR genes. It has been reported previously that EDS1 interacts with TIR domain containing R genes and NDR1 associates with CC containing R genes [7] [47] [48] [49]. Interestingly, we also got the similar results of EDS1 and NDR1 interactions with different subsets of NBS$L R R$ genes through co-expression analysis. We found that in case of PM-responsive genes, out of 6 NBS$L R R$ genes, 4 genes consisting of CC domain are interacting with NDR1 protein whereas 2 genes containing TIR domain are interacting with EDS1 proteins (Fig. 2A). Similarly, we got 7 NBS-LRR genes in response to DM infection, amongst which 4 genes having $C C$ domains were interacting with NDR1 and 3 genes consisted of TIR domain are associated with EDS1 (Fig. 2B). 
Next, as it was described above that EDS1 associates with PAD4, we got one PM-responsive PAD4 gene i.e XP_010652916.1 that is strongly interacting with EDS1 gene (NP_001267967.1) (Fig. 2A).

We also find a strong interaction of EDS1 and NDR1 genes with RAR1 gene. This protein stabilizes the NBS-LRR proteins, thus playing an immense role in ETI [50] [51] [52] [53] [54] [55]. All these proteins interacting till now leads to accumulation of SA in cell which further promotes the activation of NPR1 and NPR2 proteins. These proteins are initially present in cytoplasm, when activated with SA accumulation, it got translocated to nucleus where it interacts with various transcription factors that eventually leads to activation of various PR proteins [56]. Through co-expression analysis, we identified 2 NPR1 proteins and 1 NPR2 protein interacting with SA in response to PM-infection and 1 NPR2 protein in response to DMinfection (Fig. 2). Both NPR1 and NPR2 were reported previously to participate in SA signal transduction in plants like Arabidopsis, rice, apple, poplar etc [57] [58] [59] [60] [61] [39].

The co-expression results depicted that NPR1 and NPR2 proteins interacts with TGA, WRKY and NAC transcription factors in case of both powdery and downy mildew infection. We chose 5 each highly interacting (co-expression value 1) TFs from both PM and DM-responsive data that were shown to be interacted with various classes of PR proteins. There have been several reports that showed that NPR genes regulate the expression of $P R$ genes by interacting with various TFs. Previously, it has been shown in Arabidopsis that the PR-1 gene expression is regulated by NPR1 gene by binding to TGA transcription factors [62]. Similarly WRKY and NAC transcription factors were also found to interact with NPR1 gene in previous studies [63] [64]. The interaction of NPR proteins with several TFs finally activates various PR proteins that provides SAR to plant. We got 6 PM-responsive and 4 DM-responsive highly co-expressing PR proteins which belongs to 5 different PR protein families i.e PR1, PR2, PR5, PR10.1 and PR10.7 (Fig. 2). Altogether, we screened $24 \mathrm{PM}$-responsive and 22 DM-responsive differentially expressed genes in $V$. vinifera accessions that were found to be highly interacting amongst each other in response to SA mediated signal transduction during powdery and downy mildew stress (Fig. 2).

\section{qPCR expression analysis}

On the basis of FPKM and co-expression analysis, we selected some highly interacting defensive genes that are activated during a signaling cascade in response to fungal infection for validation by qPCR. We chose 14 PM-responsive and 13 DM-responsive genes for real time expression analysis under various stress conditions (Fig. 3). The primer sequences, amplicon length and annealing temperature of PM and DM-responsive defensive genes is listed in table S3 and table S4.

There are reports that tell us about the importance of SA during pathogenic attack. It is evident in literature through experiments performed in tobacco and Arabidopsis plants that basal and SAR signaled through R genes will be compromised if SA is not available [65] [66] [67]. Similarly, the Arabidopsis eds5 and eds 16 mutants that are lacking SA accumulation are compromised in some $R$-gene pathways as well as SAR and basal resistance [68] [69] [70]. It has been reported previously that exogenous application of SA or SA analogs induces SAR and restores resistance in numerous mutants compromised in signaling steps upstream of SA production [49] [71] [72] [73]. We also applied SA 
exogenously on leaves of Thompson seedless variety at 5 different time intervals i.e $1 \mathrm{hpi}, 2 \mathrm{hpi}, 1 \mathrm{dpi}, 2 \mathrm{dpi}$ and 3dpi to study the change in expression patterns of various defense related genes.

Consequently, we found that most of the genes got upregulated during 2dpi PM infection, 2 hpi and 3 dpi SA treatment (Fig. 3A). Similarly, for DM, genes were mostly upregulated during late fungal infection, $1 \mathrm{hpi}$ and 3 dpi SA treatment (Fig. 3B). The possible assumption would be that after fungal infection, SA levels rise in plant leading to defense reaction. So, when we gave SA treatment to our samples, initially genes got upregulated in $1 \mathrm{hr}$ or $2 \mathrm{hr}$; and later on to provide SAR to plant, the expression level peaks up at 3dpi in both fungal infections.

Additionally, our results of powdery and downy mildew infection through quantitative real time PCR are in accordance with results obtained through FPKM analysis. Most of the genes got up-regulated during PM and DM infection as compared to control condition in both FPKM and real time analysis.

\section{Chromosomal distribution of PM and DM-responsive genes}

After identification of PM and DM-responsive defensive genes obtained through FPKM and co-expression analysis studies, we finally got 24 PM-responsive and 22 DM-responsive defensive genes in the grape genome. We characterized the identified genes on the basis of chromosomal distribution which showed that PM and DM-responsive defensive genes are distributed widely in the grape genome. Amongst all, PM-responsive NBS-LRR genes were located on different 5 chromosomes i.e. 5, 11, 14, 15 and 19 (Fig. 4A). Similarly, PR proteins were distributed on chromosome 4, 5, 7, 8 and 18. EDS1 is present on chromosome 17; NDR1 on chromosome 7; PAD4 on chromosome 16 and NPR1 and NPR2 were located on chromosomes 11, 7 and 10 (Fig. 4A). Likewise, DM-responsive defensive genes were also unevenly distributed on the grape genome. Amongst all, DM-responsive NBS-LRR genes were located on chromosomes 5, 13, 14, 15 and 19 (Fig. 4B). Likewise, PR proteins were located on chromosomes 4, 7, 8 and 18; the chromosomes shared between PM and DM-responsive PR proteins are same (Fig. 4B). EDS1 genes were present on chromosome 17; NDR1 on chromosomes 9 and 10; RAR1 on chromosome 16 and NPR2 on chromosome 10 (Fig. 4B). Altogether, out of 24 PM-responsive defensive genes, maximum number (5) of genes were present on chromosome 5, followed by 4 genes on chr7, 3 and 2 genes each on chromosome numbers 11 and 18 (Fig. 4A). Similarly, for DM-responsive defensive genes, 3 genes were present on chromosome 5; 2 genes each on chromosomes 7, 10, 17 and 18 followed by a single gene number on various other chromosomes (Fig. 4B). Additionally, we identified 1 gene of DM-responsive pathway that was not placed on any scaffold.

\section{Physicochemical characteristics of PM and DM-responsive defensive genes}

The average length, molecular weight, physical index (pl) and instability index of PM-responsive defensive proteins was 467 amino acids, $526.69 \mathrm{kDa}, 6.6$ and 46.57 respectively (Table S1). We found the maximum and minimum polypeptide length to be 1413 and 112 amino acids. Likewise, the maximum and minimum molecular weight depicted was $159.516 \mathrm{kDa}$ and $17.221 \mathrm{kDa}$. The maximum pl determined was 9.45 whereas minimum was 4.84 respectively. To find out whether the protein is stable or not, we 
deduced the instability index. The maximum and minimum instability index found was 91.98 and 24.90 and out of 24, 6 such proteins were identified that were found to be stable and 18 proteins were unstable (Table S1).

Similarly, The average length, molecular weight, physical index (pl) and instability index of DM-responsive defensive proteins was 576 amino acids, $650.81 \mathrm{kDa}, 7.49$ and 46.15 respectively (Table S2). Through our analysis, we got the maximum and minimum polypeptide length to be 2130 and 136 amino acids. Similarly, the maximum and minimum molecular weight depicted was $242.899 \mathrm{kDa}$ and $15.758 \mathrm{kDa}$. The maximum pl determined was 10.19 whereas minimum was 5.06 respectively. Our results suggested that it could be easier to clone fungal-responsive defensive genes due to small size of these proteins and subsequently various transgenic studies could be done.

The values of 57.17 and 31.49 were found to be the maximum and minimum instability index. Our results suggested that out of 22, 4 such proteins were identified that were found to be stable and 18 proteins were unstable (Table S2). Most of the genes that were found stable are ones belonging to NDR1 and PR protein family. The PR protein families that were found to be stable includes PR1, PR2, PR10.1 and PR10.7.

\section{Gene structure and motif analysis}

The structural analysis of PM and DM-responsive defensive genes was depicted in terms of introns and exons that were found to be widely diverged. The three processes are known to be responsible for diversed structures of exons and introns: insertion/ deletion, exonization/pseudo-exonization and exon/intron gain/loss [74] [75] [76].

For PM-responsive defensive genes, the intron numbers were 1 to 7 with the maximum intron number of 7 in 1 gene; in contrast, the minimum intron number was one in 3 genes. The most frequent intron number in maximum sequences is 2 and 3 . Moreover, 5 such genes were identified that lacked introns (Fig. 5A). Similarly, for DM-responsive defensive genes, the intron numbers were 1 to 7 with the maximum intron number of 7 in 1 gene; in contrast, the lowest intron number was one in 2 genes. The most frequent intron number in maximum sequences was 2. We also identified 9 intron-less genes (Fig. 5B).

Next, we depicted various intron phases based on the location of intron with respect to reading frame of gene. As a result, 3 types of intron phases were found in our sequences. Mostly. the introns were in phase 0 , followed by introns in phase 1 and phase 2. For PM-responsive defensive genes, approximately, 54 of the introns were present in 0 phase, $36 \%$ in 1 st phase and $10 \%$ in 2nd phase, respectively (Fig. 5A). For DM-responsive defensive genes, approximately, $58 \%$ of the introns were present in 0 phase, $32 \%$ in $1 \mathrm{st}$ phase and $10 \%$ in 2 nd phase, respectively (Fig. 5B).

Next, the candidate protein sequences were examined for the presence of various motifs by using 'MEME' server and Pfam database. The results were further verified through InterProScan for the presence of desired motif. As a result, we identified 18 different conserved motifs dispersed throughout the protein 
sequences in PM and DM-responsive defensive genes (Fig. 5). The various conserved domains identified amongst different classes of defensive proteins includes NB-ARC and LRR8 and Rx-N in NBS-LRR proteins; Lipase 3 and EDS EP domains in EDS and PAD4 proteins; LEA2 domain in NDR1 proteins; BTB, DUF3420, Ank2, BetV1 and NPR1 interacting domains in NPR1 and NPR2 proteins; CHORD domain in RAR1 protein; bZIP1, bZIPC, NAM and WRKY in TFs; Thaumatin, Glycohydro 17, Betv1 and CAP in different classes of PR proteins. The block diagrams of the conserved motifs are shown in Fig. 5 . The presence of these motifs is necessary for defensive proteins to perform various important activities.

\section{Functional prediction of defensive genes}

To understand the diverse functions of proteins at molecular level, we performed Blast2GO that depicted the biological process, molecular function and cellular component, the 3 major characteristics of genes. Various $\mathrm{GO}$ terms were assigned to our gene sequences to determine the possible functions of PM and DM-responsive defensive genes. The results of GO terms allocations showed that maximum number of sequences were involved in biological processes; followed by sequences in cellular component and molecular function category in both PM and DM-responsive genes.

For PM-responsive genes, in biological process category, we obtained $16 \%$ sequences that were involved in response to stimulus (G0:0050896); 10\% each in biological process regulation (G0:0065007) and cellular process regulation (G0:0009987); 11\% sequences in metabolic process regulation (G0:0019222); $7 \%$ each in immune system process (G0:0002376) and signaling (GO: 0023052) etc [Supp. Fig. 1A(a)]. Likewise, the $\mathrm{GO}$ term annotations in cellular component category suggested that most of our gene sequences were performing their respective functions in cell (23\%), cell part $(23 \%)$, organelle $(20 \%)$, membrane (7\%), extracellular region (7\%), membrane part (6\%) etc. [Supp. Fig. 1A (b)] and the G0 terms assigned to these components included G0:0005623, G0:0044464, GO:0043226, G0:0016020, G0:0005576 and G0:0044425. The results of molecular process category showed that maximum number of sequences (52\%) were involved in binding activity (G0:0005488), followed by $19 \%$ in transcription regulator activity (G0:0140110), 15\% in catalytic activity (GO:0003824), 7\% each in molecular function regulator (GO:0098772) and molecular transducer activity (G0:0060089) [Supp. Fig. 1A(c)].

Similarly, for DM-responsive genes, in biological process annotations, the percentage of gene sequences involved in various processes were $11 \%$ for metabolic process, $9 \%$ for cellular process, $7 \%$ for immune system process, $10 \%$ for biological regulation, $15 \%$ in response to stimulus, $5 \%$ for signaling etc [Supp. Fig. 1B(a)]. Likewise, in cellular component category, $24 \%$ of sequences each were found to be executing their role in cell and cell part; $19 \%$ in organelle, $10 \%$ in membrane, $5 \%$ each in membrane part and extracellular region [Supp. Fig. 1B (b)]. The annotations of molecular process category depicted most of the genes to be involved in binding activity $(60 \%) ; 20 \%$ of the sequences were included in transcription regulator activity; $12 \%$ in catalytic activity, $4 \%$ each in molecular function regulator and molecular transducer activity [Supp. Fig. 1B(c)].

Altogether, our results depicted that the PM and DM-responsive defensive genes identified in our study might be playing an eminent role in biological, cellular and molecular processes. 
In response to biotic and abiotic stresses, a complex regulatory mechanism is activated in plants, therefore the characterization of PM and DM stress-responsive regulatory elements will provide an important information regarding upstream regulation of PM and DM-responsive defensive proteins. We chose an upstream sequence of upto $2 \mathrm{~kb}$ sequence from the transcription start site for identifying cis elements that are associated with stress induced gene expression in promoter regions of defensive genes. As a result of our analysis, we found 2 primary cis-acting elements i.e TATA and CAAT boxes in promoter and enhancer regions of all gene sequences. Next, we focused on the identification of PM and DM stress specific promoter elements. Consequently, 5 types of fungal stress-responsive regulatory elements namely salicylic acid responsive element (TCA element), defense and stress responsive element (TC-rich repeats), wound and pathogen responsive element (WUN-motif and W-box), stress responsive element (GT1 Box), As-1 and TGA were identified in PM and DM-responsive promoter sequences; the transcription factors binds to these cis-regulatory elements and activates the stress tolerant pathway. Amongst all, we got salicylic acid responsive element in 15 each PM-responsive and DM-responsive promoter sequences, wound and pathogen responsive element in $17 \mathrm{PM}$-responsive and $16 \mathrm{DM}$ responsive sequences, defense and stress responsive element in 12 PM-responsive and 10 DMresponsive sequences, As-1 in 10 each in PM-responsive and DM-responsive sequences and TGA motif in 6 PM-responsive and 9 DM-responsive sequences (Fig. 6). Previously, the role of various TFs have been identified in response to PM and DM-stress; NAC TFs bind to GT1 box and bZIP TFs associates with TGA elements; WRKY binds to W-box in response to fungal infection [77] [78] [79]. In our analysis, we observed the presence of these elements in defensive proteins which strongly supported that these genes got upregulated in situation of PM and DM stress as a defense mechanism. Altogether, we could say that there were various types and number of regulatory elements present in promoter regions of defensive genes involved in signaling cascade, indicating that these genes must be playing an eminent role in regulatory mechanisms in response powdery and downy mildew stress condition.

\section{Conclusion}

In our study, grapevine genome was explored to identify PM and DM-responsive defensive genes that could be used to improve disease resistance. Consequently, 47 PM-responsive and 98 DM-responsive differentially expressed genes were identified in 2 different $V$. vinifera accessions amongst which 24 PMresponsive and 22 DM-responsive defensive genes are found to be highly interacting with each other. Also, we explored the various characteristic features of the identified PM and DM-responsive defensive genes such as gene organization, motif analysis, gene regulation, chromosomal localization and functional analysis. Altogether, we can summarize that the pathogen responsive defensive genes identified in our present study could be beneficial in enhancing powdery and downy mildew resistance in grapevine.

\section{Declarations}


ETHICS APPROVAL AND CONSENT TO PARTICIPATE: Not Applicable

CONSENT FOR PUBLICATION: Not Applicable

AVAILABILITY OF DATA AND MATERIAL: Not applicable

FUNDING: SERB, India (grant number EEQ/2016/000103)

AUTHOR CONTRIBUTIONS: KS; conceived the idea, designed the experiments, analyzed the results and finalized the manuscript. N Goyal and GB, collected and analyzed the data, compiled the results. AKU provided the plant material analyzed the results. N Goyal and N Garewal performed wetlab experiments and wrote the manuscript.

ACKNOWLEDGEMENTS: Authors are thankful to SERB, India to provide research grant to carry out this work. NG and GB are thankful to DBT, India and ICMR India for providing junior and senior research fellowships.

COMPETING INTEREST: All the authors declare that there is no conflict of interest.

\section{References}

[1]C. Gessler, I. Pertot, M. Perazzolli, Plasmopara viticola: a review of knowledge on downy mildew of grapevine and effective disease management, (2011).

[2]T. Boller, Chemoperception of microbial signals in plant cells, Annu. Rev. Plant Biol. 46 (1995) 189214.

[3]A. G. Darvill, P. Albersheim, Phytoalexins and their elicitors-a defense against microbial infection in plants, Annu. Rev. Plant Physiol. 35 (1984) 243-275.

[4]G. B. Martin, A. J. Bogdanove, G. Sessa, Understanding the functions of plant disease resistance proteins, Annu. Rev. Plant Biol. 54 (2003) 23-61.

[5]Z. Nimchuk, T. Eulgem, B. F. Holt lii, J. L. Dangl, Recognition and response in the plant immune system, Annu. Rev. Genet. 37 (2003) 579-609.

[6]G. Loake, M. Grant, Salicylic acid in plant defence-the players and protagonists, Curr. Opin. Plant Biol. 10 (2007) 466-472.

[7]N. Aarts, M. Metz, E. Holub, B. J. Staskawicz, M. J. Daniels, J. E. Parker, Different requirements for EDS1 and NDR1 by disease resistance genes define at least two R gene-mediated signaling pathways in Arabidopsis, Proc. Natl. Acad. Sci. 95 (1998) 10306-10311.

[8]K. Heidrich, L. Wirthmueller, C. Tasset, C. Pouzet, L. Deslandes, J. E. Parker, Arabidopsis EDS1 connects pathogen effector recognition to cell compartment-specific immune responses, Science (80-.). 334 
(2011) 1401-1404.

[9]D. Selote, M. B. Shine, G. P. Robin, A. Kachroo, Soybean NDR 1-like proteins bind pathogen effectors and regulate resistance signaling, New Phytol. 202 (2014) 485-498.

[10]J. Wang, M. B. Shine, Q.-M. Gao, D. Navarre, W. Jiang, C. Liu, Q. Chen, G. Hu, A. Kachroo, Enhanced disease susceptibility 1 mediates pathogen resistance and virulence function of a bacterial effector in soybean, Plant Physiol. 165 (2014) 1269-1284.

[11]S. Bhattacharjee, M. K. Halane, S. H. Kim, W. Gassmann, Pathogen effectors target Arabidopsis EDS1 and alter its interactions with immune regulators, Science (80-.). 334 (2011) 1405-1408.

[12]W. E. Durrant, X. Dong, Systemic acquired resistance, Annu. Rev. Phytopathol. 42 (2004) 185-209.

[13]Z. Mou, W. Fan, X. Dong, Inducers of plant systemic acquired resistance regulate NPR1 function through redox changes, Cell. 113 (2003) 935-944.

[14]T. Eulgem, I. E. Somssich, Networks of WRKY transcription factors in defense signaling, Curr. Opin. Plant Biol. 10 (2007) 366-371.

[15]X. Dong, NPR1, all things considered, Curr. Opin. Plant Biol. 7 (2004) 547-552.

[16]S. L. Toffolatti, G. De Lorenzis, A. Costa, G. Maddalena, A. Passera, M. C. Bonza, M. Pindo, E. Stefani, A. Cestaro, P. Casati, Unique resistance traits against downy mildew from the center of origin of grapevine (Vitis vinifera), Sci. Rep. 8 (2018) 12523.

[17]N. Goyal, G. Bhatia, S. Sharma, N. Garewal, A. Upadhyay, S. K. Upadhyay, K. Singh, Genome-wide characterization revealed role of NBS-LRR genes during powdery mildew infection in Vitis vinifera, Genomics. (2019).

[18]B. J. Haas, A. Papanicolaou, M. Yassour, M. Grabherr, P. D. Blood, J. Bowden, M. B. Couger, D. Eccles, B. Li, M. Lieber, De novo transcript sequence reconstruction from RNA-seq using the Trinity platform for reference generation and analysis, Nat. Protoc. 8 (2013) 1494.

[19]J. Seo, H. Gordish-Dressman, E. P. Hoffman, An interactive power analysis tool for microarray hypothesis testing and generation, Bioinformatics. 22 (2006) 808-814.

[20]S. Ghawana, A. Paul, H. Kumar, A. Kumar, H. Singh, P. K. Bhardwaj, A. Rani, R. S. Singh, J. Raizada, K. Singh, An RNA isolation system for plant tissues rich in secondary metabolites, BMC Res. Notes. 4 (2011) 85 .

[21]K. J. Livak, T. D. Schmittgen, Analysis of relative gene expression data using real-time quantitative PCR and the 2- $\triangle \Delta C T$ method, Methods. 25 (2001) 402-408. 
[22]M. W. Pfaffl, G. W. Horgan, L. Dempfle, Relative expression software tool (REST@) for group-wise comparison and statistical analysis of relative expression results in real-time PCR, Nucleic Acids Res. 30 (2002) e36-e36.

[23]B. Hu, J. Jin, A.-Y. Guo, H. Zhang, J. Luo, G. Gao, GSDS 2.0: an upgraded gene feature visualization server, Bioinformatics. 31 (2014) 1296-1297.

[24]T. L. Bailey, C. Elkan, Fitting a mixture model by expectation maximization to discover motifs in bipolymers, (1994).

[25]A. Conesa, S. Götz, Blast2GO: A comprehensive suite for functional analysis in plant genomics, Int. J. Plant Genomics. 2008 (2008).

[26]M. Lescot, P. Déhais, G. Thijs, K. Marchal, Y. Moreau, Y. Van de Peer, P. Rouzé, S. Rombauts, PlantCARE, a database of plant cis-acting regulatory elements and a portal to tools for in silico analysis of promoter sequences, Nucleic Acids Res. 30 (2002) 325-327.

[27]E. Gasteiger, C. Hoogland, A. Gattiker, M. R. Wilkins, R. D. Appel, A. Bairoch, Protein identification and analysis tools on the ExPASy server, in: Proteomics Protoc. Handb., Springer, 2005: pp. 571-607.

[28]F. Dunemann, A. Peil, A. Urbanietz, T. Garcia-Libreros, Mapping of the apple powdery mildew resistance gene PI1 and its genetic association with an NBS-LRR candidate resistance gene, Plant Breed. 126 (2007) 476-481.

[29]S. Xiao, S. Ellwood, O. Calis, E. Patrick, T. Li, M. Coleman, J. G. Turner, Broad-spectrum mildew resistance in Arabidopsis thaliana mediated by RPW8, Science (80-.). 291 (2001) 118-120.

[30]P. D. Bittner-Eddy, I. R. Crute, E. B. Holub, J. L. Beynon, RPP13 is a simple locus in Arabidopsis thaliana for alleles that specify downy mildew resistance to different avirulence determinants in Peronospora parasitica, Plant J. 21 (2000) 177-188.

[31]H. Zhang, H. Guan, J. Li, J. Zhu, C. Xie, Y. Zhou, X. Duan, T. Yang, Q. Sun, Z. Liu, Genetic and comparative genomics mapping reveals that a powdery mildew resistance gene MI3D232 originating from wild emmer co-segregates with an NBS-LRR analog in common wheat (Triticum aestivum L.), Theor. Appl. Genet. 121 (2010) 1613-1621.

[32]C. Coleman, D. Copetti, G. Cipriani, S. Hoffmann, P. Kozma, L. Kovács, M. Morgante, R. Testolin, G. Di Gaspero, The powdery mildew resistance gene REN1 co-segregates with an NBS-LRR gene cluster in two Central Asian grapevines, BMC Genet. 10 (2009) 89.

[33]G. Di Gaspero, G. Cipriani, Resistance gene analogs are candidate markers for disease-resistance genes in grape (Vitis spp.), Theor. Appl. Genet. 106 (2002) 163-172. 
[34]H. Wan, Z. Zhao, A. A. Malik, C. Qian, J. Chen, Identification and characterization of potential NBSencoding resistance genes and induction kinetics of a putative candidate gene associated with downy mildew resistance in Cucumis, BMC Plant Biol. 10 (2010) 186.

[35]B. J. Feys, L. J. Moisan, M. Newman, J. E. Parker, Direct interaction between the Arabidopsis disease resistance signaling proteins, EDS1 and PAD4, EMBO J. 20 (2001) 5400-5411.

[36]H. A. Fitzgerald, M.-S. Chern, R. Navarre, P. C. Ronald, Overexpression of (At) NPR1 in rice leads to a BTH-and environment-induced lesion-mimic/cell death phenotype, Mol. Plant-Microbe Interact. 17 (2004) $140-151$.

[37]R. Makandar, J. S. Essig, M. A. Schapaugh, H. N. Trick, J. Shah, Genetically engineered resistance to Fusarium head blight in wheat by expression of Arabidopsis NPR1, Mol. Plant-Microbe Interact. 19 (2006) 123-129.

[38]W.-C. Lin, C.-F. Lu, J.-W. Wu, M.-L. Cheng, Y.-M. Lin, N.-S. Yang, L. Black, S. K. Green, J.-F. Wang, C.-P. Cheng, Transgenic tomato plants expressing the Arabidopsis NPR1 gene display enhanced resistance to a spectrum of fungal and bacterial diseases, Transgenic Res. 13 (2004) 567-581.

[39]M. Malnoy, Q. Jin, E. E. Borejsza-Wysocka, S. Y. He, H. S. Aldwinckle, Overexpression of the apple MpNPR1 gene confers increased disease resistance in Malus× domestica, Mol. Plant-Microbe Interact. 20 (2007) 1568-1580.

[40]L. C. Van Loon, W. S. Pierpoint, T. H. Boller, V. Conejero, Recommendations for naming plant pathogenesis-related proteins, Plant Mol. Biol. Report. 12 (1994) 245-264.

[41]L. C. Van Loon, E. A. Van Strien, The families of pathogenesis-related proteins, their activities, and comparative analysis of PR-1 type proteins, Physiol. Mol. Plant Pathol. 55 (1999) 85-97.

[42]B. Walter, D. Joly, C. Bertsch, Sequence of a putative Vitis vinifera PR-1, Vitis J. Grapevine Res. 42 (2003) 103-104.

[43]A. K. Jacobs, I. B. Dry, S. P. Robinson, Induction of different pathogenesis-related cDNAs in grapevine infected with powdery mildew and treated with ethephon., Plant Pathol. 48 (1999) 325-336.

[44]W. K. Roberts, C. P. Selitrennikoff, Zeamatin, an antifungal protein from maize with membranepermeabilizing activity, Microbiology. 136 (1990) 1771-1778.

[45]J.-J. Liu, A. K. M. Ekramoddoullah, The family 10 of plant pathogenesis-related proteins: their structure, regulation, and function in response to biotic and abiotic stresses, Physiol. Mol. Plant Pathol. 68 (2006) 3-13.

[46]M. He, Y. Xu, J. Cao, Z. Zhu, Y. Jiao, Y. Wang, X. Guan, Y. Yang, W. Xu, Z. Fu, Subcellular localization and functional analyses of a PR10 protein gene from Vitis pseudoreticulata in response to Plasmopara 
viticola infection, Protoplasma. 250 (2013) 129-140.

[47]A. Falk, B. J. Feys, L. N. Frost, J. D. G. Jones, M. J. Daniels, J. E. Parker, EDS1, an essential component of $\mathrm{R}$ gene-mediated disease resistance in Arabidopsis has homology to eukaryotic lipases, Proc. Natl. Acad. Sci. 96 (1999) 3292-3297.

[48]L. Wirthmueller, Y. Zhang, J. D. G. Jones, J. E. Parker, Nuclear accumulation of the Arabidopsis immune receptor RPS4 is necessary for triggering EDS1-dependent defense, Curr. Biol. 17 (2007) 20232029.

[49]K. S. Century, E. B. Holub, B. J. Staskawicz, NDR1, a locus of Arabidopsis thaliana that is required for disease resistance to both a bacterial and a fungal pathogen, Proc. Natl. Acad. Sci. 92 (1995) 65976601.

[50]A. Takahashi, C. Casais, K. Ichimura, K. Shirasu, HSP90 interacts with RAR1 and SGT1 and is essential for RPS2-mediated disease resistance in Arabidopsis, Proc. Natl. Acad. Sci. 100 (2003) 1177711782.

[51]D. A. Hubert, P. Tornero, Y. Belkhadir, P. Krishna, A. Takahashi, K. Shirasu, J. L. Dangl, Cytosolic HSP90 associates with and modulates the Arabidopsis RPM1 disease resistance protein, EMBO J. 22 (2003) 5679-5689.

[52]R. Lu, I. Malcuit, P. Moffett, M. T. Ruiz, J. Peart, A. Wu, J. P. Rathjen, A. Bendahmane, L. Day, D.C. Baulcombe, High throughput virus-induced gene silencing implicates heat shock protein 90 in plant disease resistance, EMBO J. 22 (2003) 5690-5699.

[53]S. Bieri, S. Mauch, Q.-H. Shen, J. Peart, A. Devoto, C. Casais, F. Ceron, S. Schulze, H.-H. Steinbiß, K. Shirasu, RAR1 positively controls steady state levels of barley MLA resistance proteins and enables sufficient MLA6 accumulation for effective resistance, Plant Cell. 16 (2004) 3480-3495.

[54]B. F. Holt, Y. Belkhadir, J. L. Dangl, Antagonistic control of disease resistance protein stability in the plant immune system, Science (80-.). 309 (2005) 929-932.

[55]C. Azevedo, S. Betsuyaku, J. Peart, A. Takahashi, L. Noel, A. Sadanandom, C. Casais, J. Parker, K. Shirasu, Role of SGT1 in resistance protein accumulation in plant immunity, EMBO J. 25 (2006) 20072016.

[56]M. Kinkema, W. Fan, X. Dong, Nuclear localization of NPR1 is required for activation of PR gene expression, Plant Cell. 12 (2000) 2339-2350.

[57]H. Cao, J. Glazebrook, J. D. Clarke, S. Volko, X. Dong, The Arabidopsis NPR1 gene that controls systemic acquired resistance encodes a novel protein containing ankyrin repeats, Cell. 88 (1997) 57-63. 
[58]S. R. Hepworth, Y. Zhang, S. McKim, X. Li, G. W. Haughn, BLADE-ON-PETIOLE-dependent signaling controls leaf and floral patterning in Arabidopsis, Plant Cell. 17 (2005) 1434-1448.

[59]G. Liu, E. B. Holub, J. M. Alonso, J. R. Ecker, P. R. Fobert, An Arabidopsis NPR1-like gene, NPR4, is required for disease resistance, Plant J. 41 (2005) 304-318.

[60]S. A. Goff, D. Ricke, T.-H. Lan, G. Presting, R. Wang, M. Dunn, J. Glazebrook, A. Sessions, P. Oeller, H. Varma, A draft sequence of the rice genome (Oryza sativa L. ssp. japonica), Science (80-.). 296 (2002) 92-100.

[61]G. A. Tuskan, S. Difazio, S. Jansson, J. Bohlmann, I. Grigoriev, U. Hellsten, N. Putnam, S. Ralph, S. Rombauts, A. Salamov, The genome of black cottonwood, Populus trichocarpa (Torr. \& Gray), Science (80-.). 313 (2006) 1596-1604.

[62]J.-M. Zhou, Y. Trifa, H. Silva, D. Pontier, E. Lam, J. Shah, D. F. Klessig, NPR1 differentially interacts with members of the TGA/OBF family of transcription factors that bind an element of the PR-1 gene required for induction by salicylic acid, Mol. Plant-Microbe Interact. 13 (2000) 191-202.

[63]S. Puranik, P. P. Sahu, P. S. Srivastava, M. Prasad, NAC proteins: regulation and role in stress tolerance, Trends Plant Sci. 17 (2012) 369-381.

[64]K. B. Singh, R. C. Foley, L. Oñate-Sánchez, Transcription factors in plant defense and stress responses, Curr. Opin. Plant Biol. 5 (2002) 430-436.

[65]T. P. Delaney, S. Uknes, B. Vernooij, L. Friedrich, K. Weymann, D. Negrotto, T. Gaffney, M. Gut-Rella, H. Kessmann, E. Ward, A central role of salicylic acid in plant disease resistance, Science (80-.). 266 (1994) $1247-1250$.

[66]T. Gaffney, L. Friedrich, B. Vernooij, D. Negrotto, G. Nye, S. Uknes, E. Ward, H. Kessmann, J. Ryals, Requirement of salicylic acid for the induction of systemic acquired resistance, Science (80-.). 261 (1993) 754-756.

[67]K. Lawton, K. Weymann, L. Friedrich, B. Vernooij, S. Uknes, J. Ryals, Systemic acquired resistance in Arabidopsis requires salicylic acid but not ethylene, MPMI-Molecular Plant Microbe Interact. 8 (1995) 863-870.

[68]J. Dewdney, T. L. Reuber, M. C. Wildermuth, A. Devoto, J. Cui, L. M. Stutius, E. P. Drummond, F. M. Ausubel, Three unique mutants of Arabidopsis identify eds loci required for limiting growth of a biotrophic fungal pathogen, Plant J. 24 (2000) 205-218.

[69]C. Nawrath, S. Heck, N. Parinthawong, J.-P. Métraux, EDS5, an essential component of salicylic aciddependent signaling for disease resistance in Arabidopsis, is a member of the MATE transporter family, Plant Cell. 14 (2002) 275-286. 
[70]M. C. Wildermuth, J. Dewdney, G. Wu, F. M. Ausubel, Isochorismate synthase is required to synthesize salicylic acid for plant defence, Nature. 414 (2001) 562.

[71]J. E. Parker, E. B. Holub, L. N. Frost, A. Falk, N. D. Gunn, M. J. Daniels, Characterization of eds1, a mutation in Arabidopsis suppressing resistance to Peronospora parasitica specified by several different RPP genes., Plant Cell. 8 (1996) 2033-2046.

[72]S. Uknes, B. Mauch-Mani, M. Moyer, S. Potter, S. Williams, S. Dincher, D. Chandler, A. Slusarenko, E. Ward, J. Ryals, Acquired resistance in Arabidopsis., Plant Cell. 4 (1992) 645-656.

[73]E. R. Ward, S. J. Uknes, S. C. Williams, S. S. Dincher, D. L. Wiederhold, D.C. Alexander, P. Ahl-Goy, J.-P. Métraux, J. A. Ryals, Coordinate gene activity in response to agents that induce systemic acquired resistance., Plant Cell. 3 (1991) 1085-1094.

[74]S. W. Roy, W. Gilbert, Rates of intron loss and gain: implications for early eukaryotic evolution, Proc. Natl. Acad. Sci. 102 (2005) 5773-5778.

[75]M. Long, N. W. VanKuren, S. Chen, M. D. Vibranovski, New gene evolution: little did we know, Annu. Rev. Genet. 47 (2013) 307-333.

[76]G. Xu, C. Guo, H. Shan, H. Kong, Divergence of duplicate genes in exon-intron structure, Proc. Natl. Acad. Sci. 109 (2012) 1187-1192.

[77]S. A. Gebrie, Promoter Analysis of a Powdery Mildew Induced Vitis vinifera NAC Transcription Factor Gene, Adv Crop Sci Tech. 5 (2017) 2.

[78]H. S. Kim, T. P. Delaney, Over-expression of TGA5, which encodes a bZIP transcription factor that interacts with NIM1/NPR1, confers SAR-independent resistance in Arabidopsis thaliana to Peronospora parasitica, Plant J. 32 (2002) 151-163.

[79]M. Wang, A. Vannozzi, G. Wang, Y.-H. Liang, G. B. Tornielli, S. Zenoni, E. Cavallini, M. Pezzotti, Z.-M. M. Cheng, Genome and transcriptome analysis of the grapevine (Vitis vinifera L.) WRKY gene family, Hortic. Res. 1 (2014) 14016.

\section{Figures}



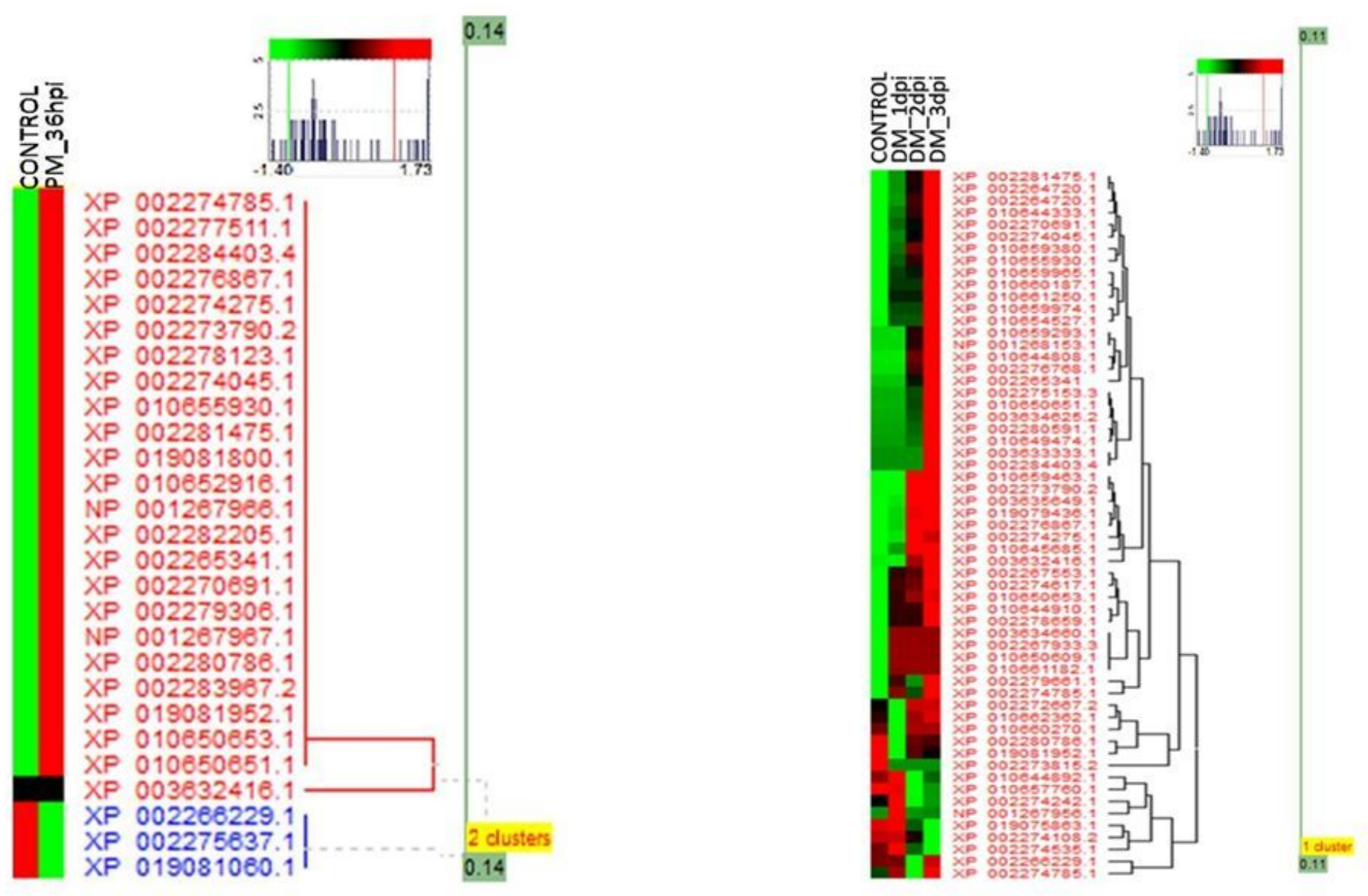

\section{Figure 1}

Heat maps representing expression levels of various defensive genes in Vitis vinfera. (a) PM-responsive defensive genes at time points of $0 \mathrm{hr}$ and $36 \mathrm{hpi}$. (b) DM-responsive defensive genes in at time points of Ohr, $1 \mathrm{dpi}$, 2dpi and $3 \mathrm{dpi}$. By increasing the brightness of green and red colours, the declined and elevated expression levels were indicated. 
(A)

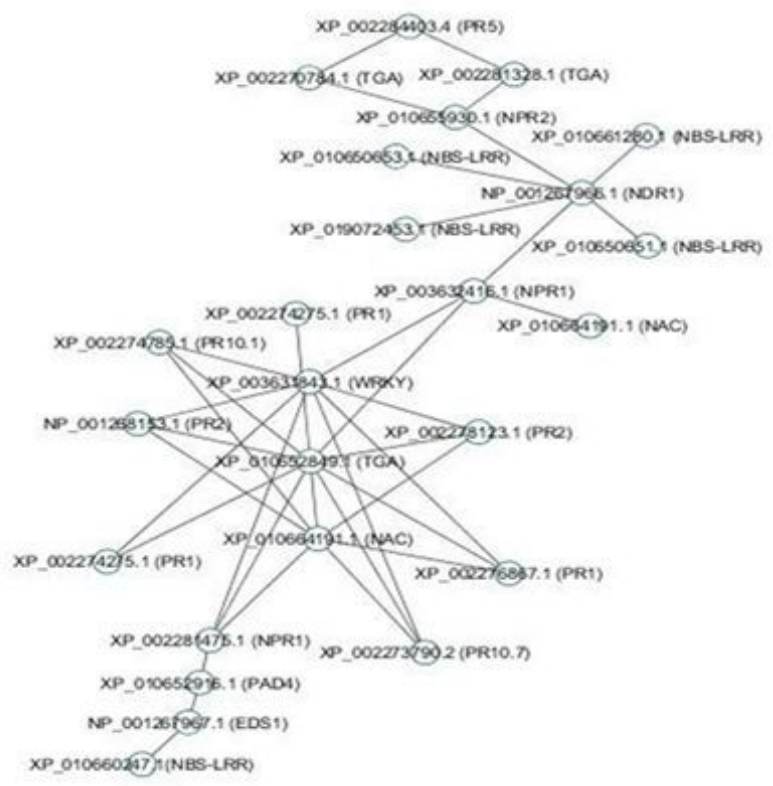

(B)

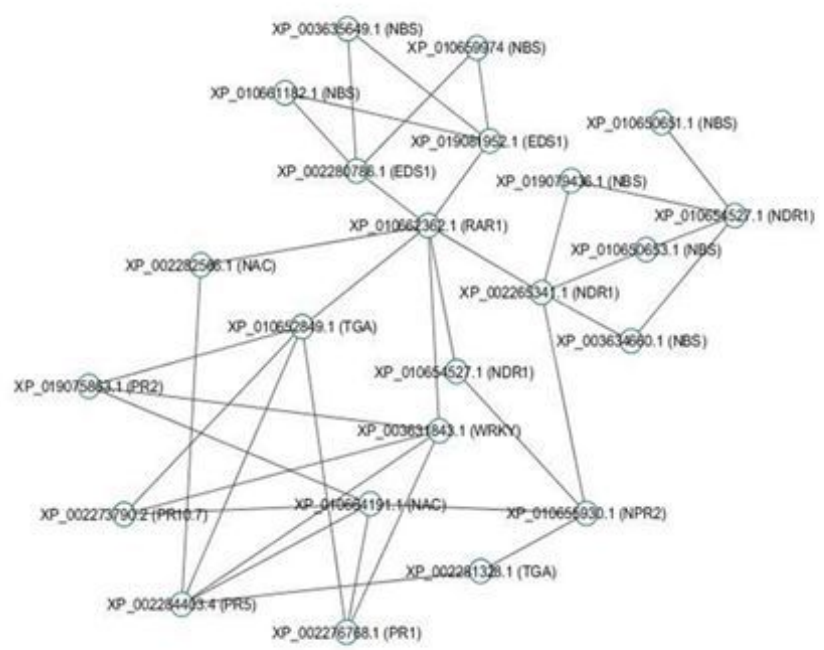

\section{Figure 2}

Co-expression analysis of defensive genes showing interaction of different types of proteins (NBS-LRR, EDS1, PAD4, NPR1, WRKY, TGA, PR) involved in signaling cascade using CoExpress 1.5.2 software. (a.) Interaction amongst PM-responsive defensive genes visualized by cytoscape 3.7.1. (b.) Interaction amongst DM-responsive defensive genes was shown. 
(A)

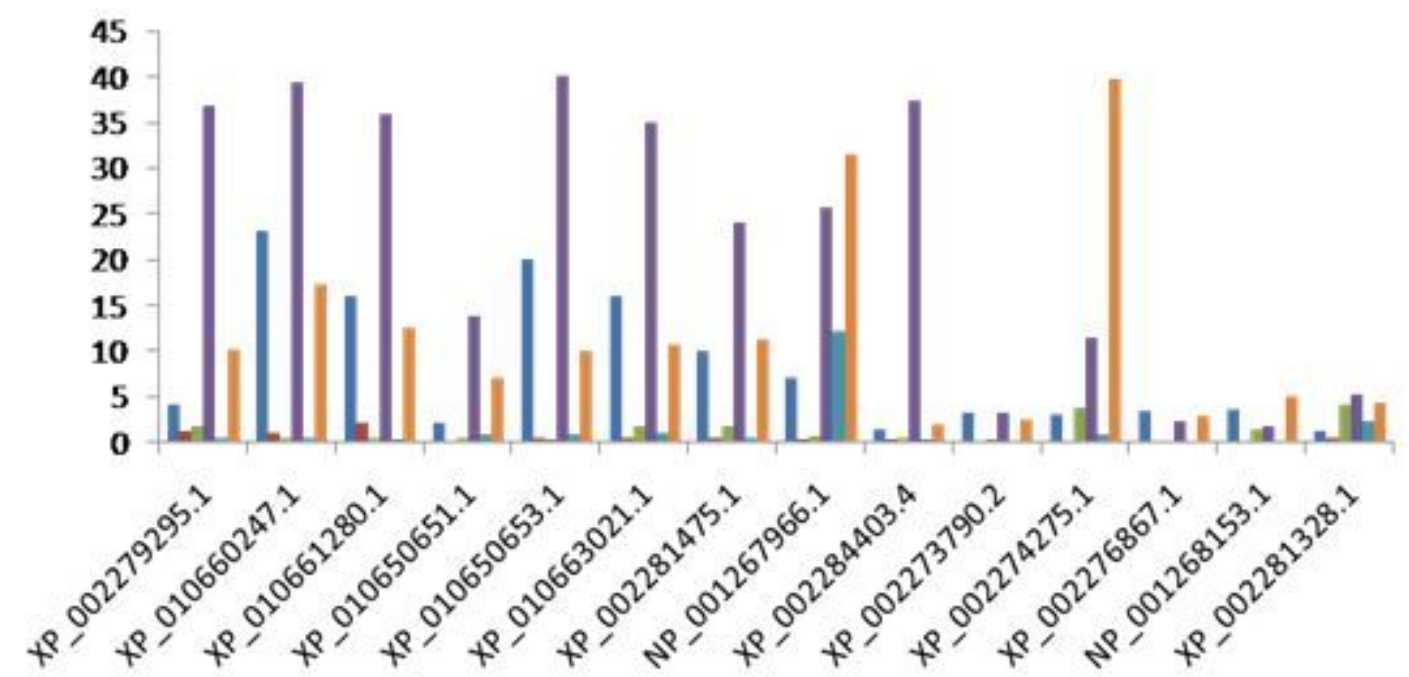

2dpi (PM infected)

1dpi(SA treated)

2dpi (SA treated)

3dpi (SA treated)

$1 \mathrm{hr}$ (SA treated)

= $2 \mathrm{hr}$ ((SA treated)

(B)

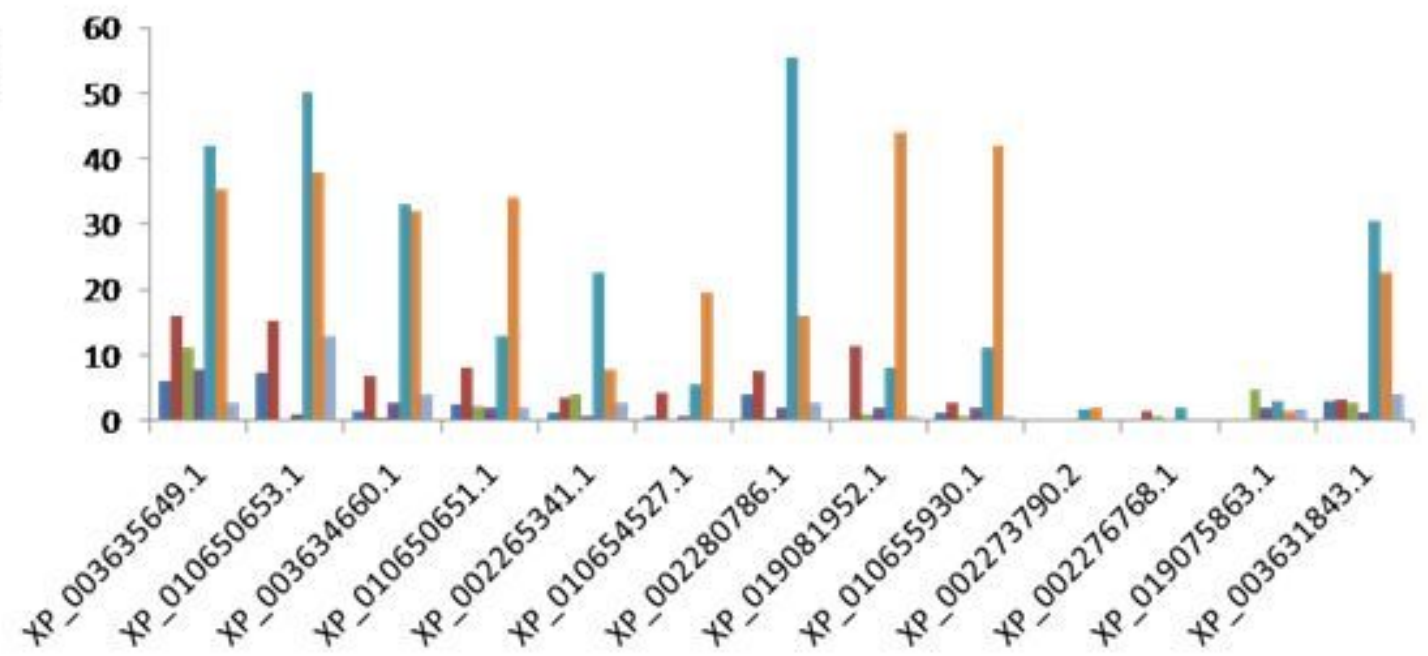

E.I (DM infected)

E.I (DM infected)

1dpi (SA treated)

2dpi (SA treated)

= 3dpi (SA treated)

=1hr (SA treated)

E $2 \mathrm{hr}$ (SA treated)

Figure 3

Relative expression levels of defensive genes through quantitative real time PCR under various stress conditions of PM and DM infection and salicylic acid (SA) treatment. (a.) Real time graph of PMresponsive defensive genes during PM infection and salicylic acid (SA) treatment. (b.) Real time graph of DM-responsive defensive genes during DM infection and salicylic acid (SA) treatment. 
(A)

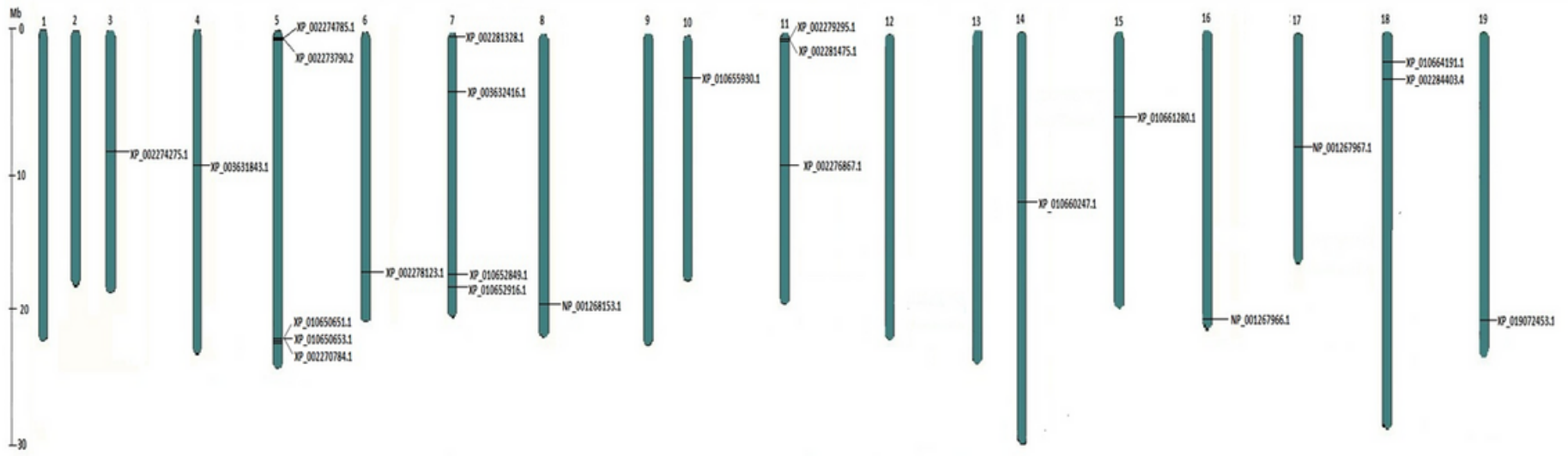

(B)
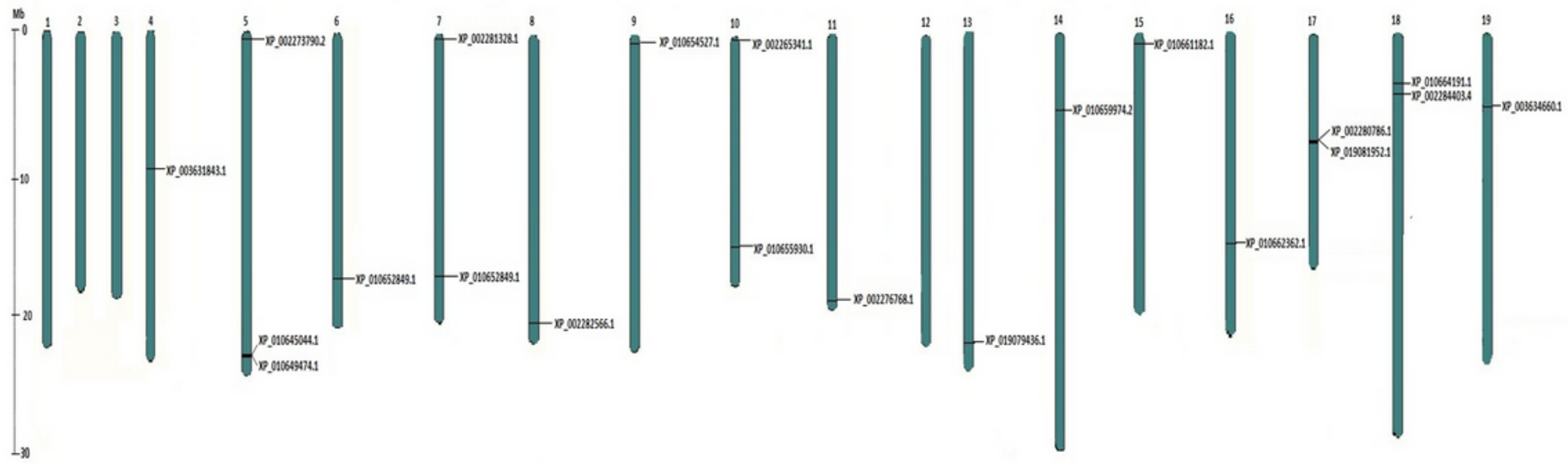

Figure 4

Chromosomal distribution of fungal-responsive defensive genes on 19 chromosomes of V. vinifera. (a.) Chromosomal locations of PM-responsive defensive genes. (b.) Chromosomal mapping of DMresponsive defensive genes. The chromosomal position of defensive genes was shown by black lines; chromosome number are exhibited on the top of chromosome and the size of chromosome is shown by vertical line. 
(A)

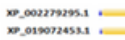

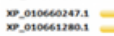

orossoes a

D.

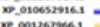

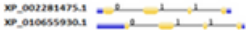

0 o

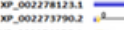

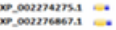

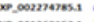

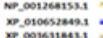

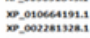

-

uened

Lesend

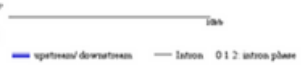

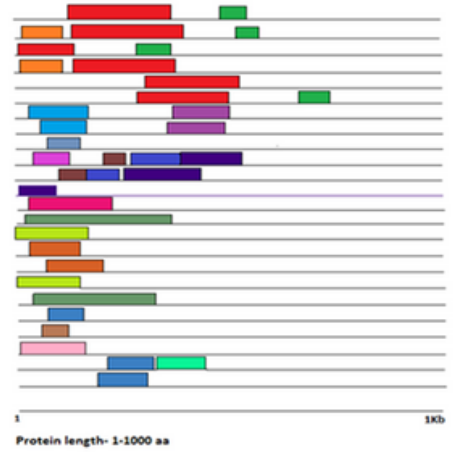
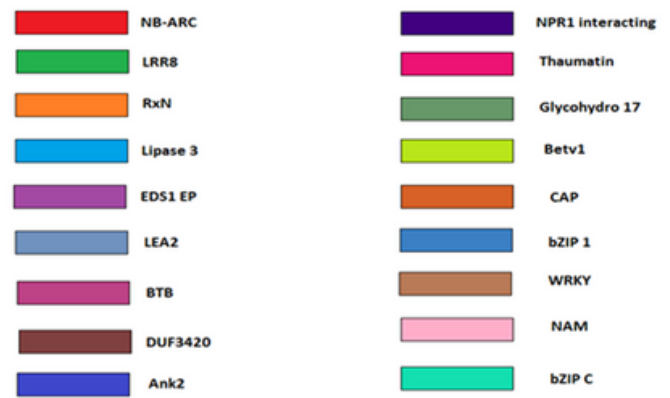

(B)

XP_010650651.1.

XP_003635649.1

XP_010650653.1

XP_019079436.1

$X P \_003634660.1$.

XP $-010659974.2=1$

XP 010661182.1

$X P$ - 010654527.1

XP_002280786.1

$X P_{-} 019081952.1$

XP 010662362.1 - i 2

XP_010655930.1 =

$x p-002284403.4$ L

$x P^{-002276768.1}$

$x p-019075863.1$

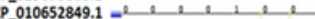

XP_003631843.1

XP_010664191.1 _ -

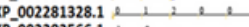

_002282566.1

$\log \cos$
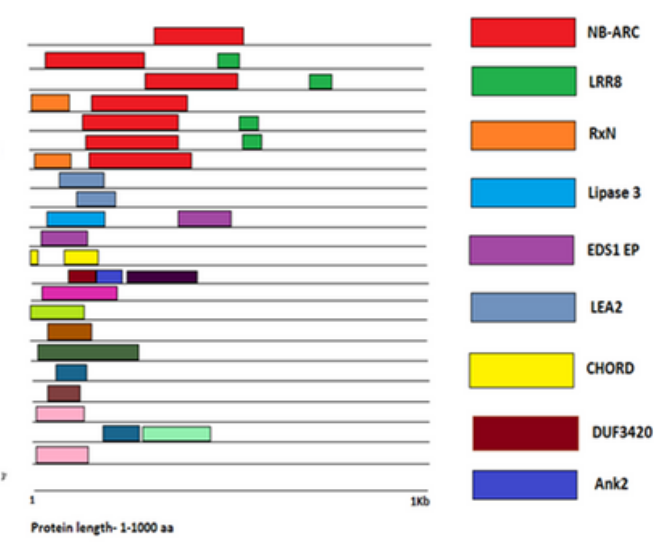

Figure 5

Gene structure and motif analysis of fungal-responsive defensive genes. The organization of introns and exons is represented by GSDS 2.0 server and motif analysis was done by using Pfam database and MEME motif based sequence analysis tool (a.) Gene structure and conserved domains of PM-responsive defensive genes. (b.) Gene structure and conserved domains of DM-responsive defensive genes. Exons are indicated by yellow boxes that are separated by thin lines of introns and UTRs are represented by blue boxes. Conserved motifs for both PM and DM-responsive genes was shown with different colored boxes. 
(A)

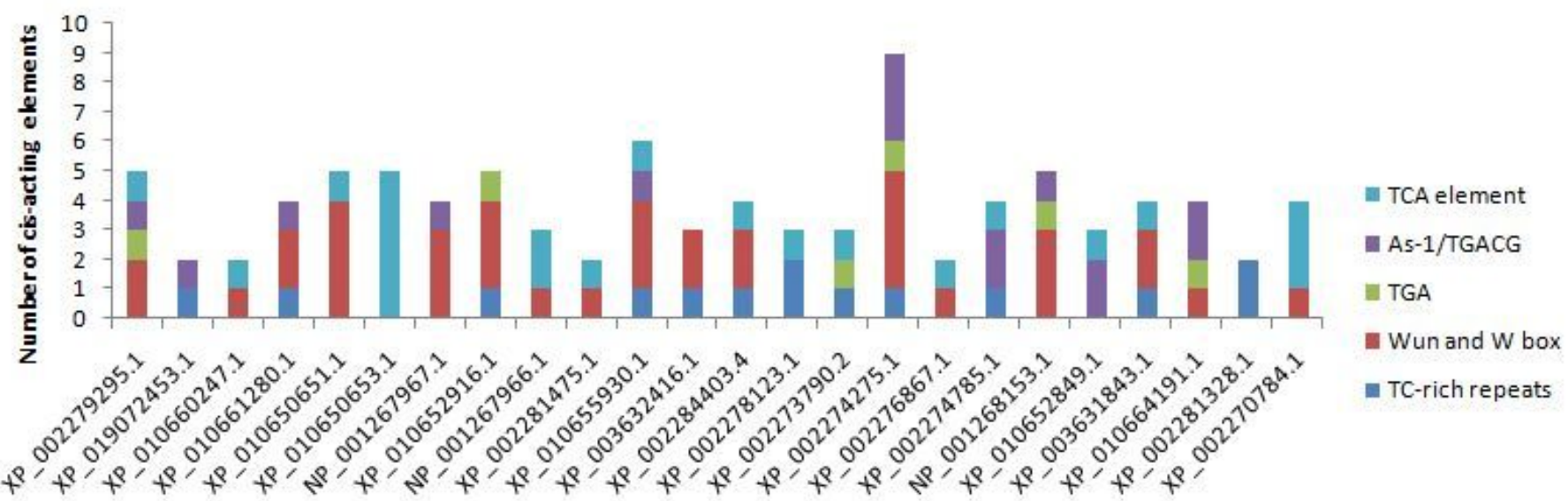

Gene I.D

(B)

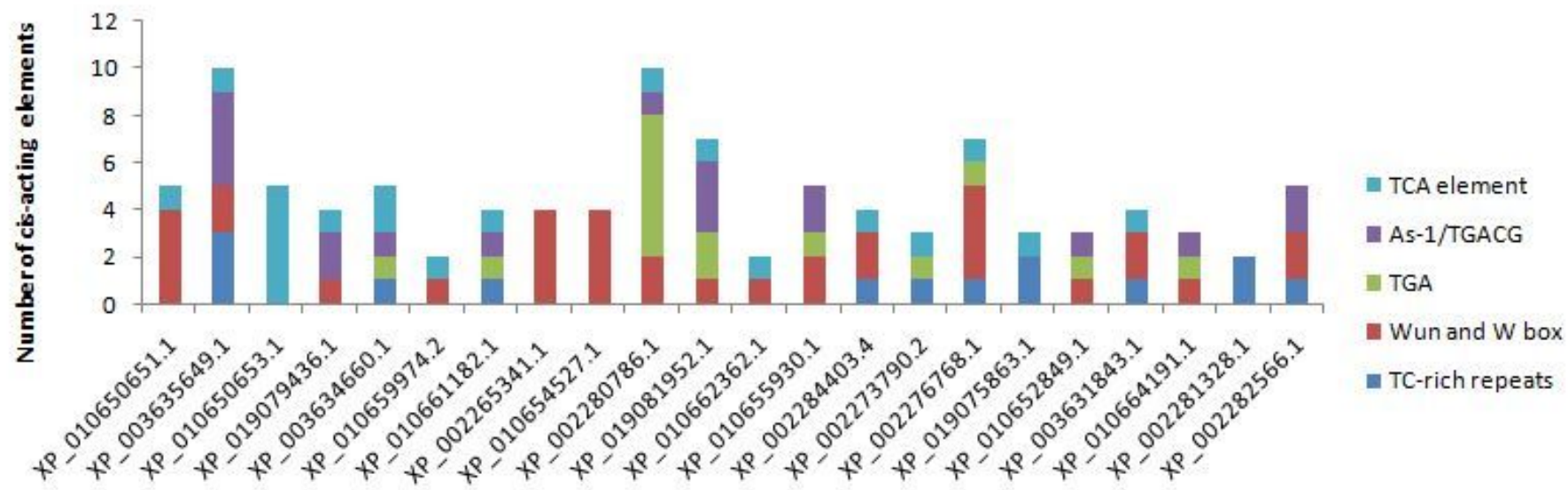

Gene I.D

\section{Figure 6}

Promoter analysis of fungal stress responsive defensive genes. (a.) PM-stress responsive (b.) DM-stress responsive cis elements were shown in different colors. Each color represents different cis-element.

\section{Supplementary Files}

This is a list of supplementary files associated with this preprint. Click to download.

- SupplFigS1.jpg

- SupplementaryTableS1.docx

- SupplementaryTableS3.docx

- SupplementaryTableS2.docx

- SupplementaryTableS4.docx 